\title{
Adult stem cell and mesenchymal progenitor theories of aging
}

\author{
So-ichiro Fukada ${ }^{1 *}$, Yuran Ma $^{1}$ and Akiyoshi Uezumi ${ }^{2}$ \\ Laboratory of Molecular and Cellular Physiology, Graduate School of Pharmaceutical Sciences, Osaka University, Osaka, Japan \\ 2 Division for Therapies Against Intractable Diseases, Institute for Comprehensive Medical Science, Fujita Health University, Toyoake, Japan
}

Edited by:

Masatake Osawa, Gifu University, Japan

Reviewed by:

Alessandra Sacco, Sanford-Burnham

Medical Research Institute, USA

Masatake Osawa, Gifu University, Japan

Julie Fradette, Centre LOEX de

I'Université Laval, CHU de Québec,

Canada

*Correspondence:

So-ichiro Fukada, Laboratory of

Molecular and Cellular Physiology,

Graduate School of Pharmaceutical

Sciences, Osaka University, 1-6

Yamada-oka, Suita, Osaka 565-0871,

Japan

e-mail: fukada@phs.osaka-u.ac.jp
Advances in medical science and technology allow people live longer lives, which results in age-related problems. Humans cannot avoid the various aged-related alterations of aging; in other words, humans cannot remain young at molecular and cellular levels. In 1956, Harman proposed the "free radical theory of aging" to explain the molecular mechanisms of aging. Telomere length, and accumulation of DNA or mitochondrial damage are also considered to be mechanisms of aging. On the other hand, stem cells are essential for maintaining tissue homeostasis by replacing parenchymal cells; therefore, the stem cell theory of aging is also used to explain the progress of aging. Importantly, the stem cell theory of aging is likely related to other theories. In addition, recent studies have started to reveal the essential roles of tissue-resident mesenchymal progenitors/stem cells/stromal cells in maintaining tissue homeostasis, and some evidence of their fundamental roles in the progression of aging has been presented. In this review, we discuss how stem cell and other theories connect to explain the progress of aging. In addition, we consider the mesenchymal progenitor theory of aging to describing the process of aging.

Keywords: aging, mesenchymal progenitors, adult stem cells, oxidative stress, telomere, DNA damage, niche

\section{INTRODUCTION}

Several theories to explain the aging-related alterations in our bodies have been proposed and accepted. The free radical theory of aging was first proposed by Harman in 1956 as one factor of aging (Harman, 1956). Aging is an universal phenomenon in living beings, and is dependent on species. Dr. Harman considered that the universality of this phenomenon meant it was caused by the same basic mechanism in all living organisms, and proposed free radicals as the causative factor. Now, the correlations between telomere length, accumulation of DNA damage, and mitochondria dysfunctions are also considered to be causative factors in aging-related alterations. Impairments of stem cell function are also supposed to explain the mechanism because stem cells are essential for maintaining tissue homeostasis, and loss of their function and/or number leads to a breakdown of function in each organ. In fact, impairments of various tissue resident stem cells during the aging process have been reported. For example, aged hematopoietic stem cells lose their engraftment potential and skew toward differentiation into myeloid cells in rodents and humans (Morrison et al., 1996; Linton and Dorshkind, 2004; Pang et al., 2011). The number of muscle stem cells (known as satellite cells) is decreased in aged mice and humans (Kadi et al., 2004; Collins et al., 2007), and some studies have indicated impaired proliferation of old satellite cells compared with young satellite cells (Bortoli et al., 2003; Conboy et al., 2005). Graying hair is a clear sign of aging in humans and many long-lived mammals (Nishimura, 2011). Melanocytes contain tyrosinase which converts tyrosine to melanin pigments in the hair during each hair cycle, but melanocyte stem cells in aged mice exhibit ectopic pigmentation or differentiation in their niche (Nishimura et al.,
2005). Although accumulating evidence has demonstrated that alterations of stem cells occur during the aging process, both intrinsic and extrinsic cellular factors in the aging of adult stem cells seem to be essential in age-related phenomena (Brack et al., 2007; Pan et al., 2007).

All adult stem cells exist in a unique microenvironment, which is known as a niche. The niche is consistent with heterogeneous types of cells and extracellular matrix proteins. The blood vessel has been proposed as a niche in common. Using expression patterns of cell surface receptors of the SLAM (signaling lymphocyte activation molecule) family in hematopoietic stem cells, Kiel et al. showed that many hematopoietic stem cells are associated with sinusoidal endothelium in the spleen and bone marrow (Kiel et al., 2005). It has been reported that neural stem cells, muscle stem cells, and spermatogonic stem cells are closely associated with blood vessels (Christov et al., 2007; Yoshida et al., 2007; Tavazoie et al., 2008). In addition to blood vessels, mesenchymal progenitors (including mesenchymal stem cell and stromal cells) have recently attracted the attention of researchers in many fields. Mesenchymal progenitors are defined as cells having potential for multipotent differentiation into mesenchymal lineages including adipocytes, osteogenic, and/or chondrogenic cells. In hematopoietic stem cells, mesenchymal progenitors also serve as one of the niche cells (Omatsu et al., 2010). When mesenchymal progenitors are depleted, the number and cell size of hematopoietic stem cells are decreased. In addition, their gene expression patterns are similar to those of the phenotype of wild hematopoietic stem cells cultured without a niche. Skeletal muscle also has muscle-resident mesenchymal progenitors, which were identified as the original source of cells of pathogenic conditions such as the accumulation 
of adipocytes and fibrosis in muscular dystrophy (Joe et al., 2010; Uezumi et al., 2010, 2011). Although the role of mesenchymal progenitors as a niche for muscle stem cells is unknown in a steady homeostatic condition, the depletion of mesenchymal progenitors in both bone marrow and skeletal muscle leads to a loss of hematopoiesis and skeletal muscle mass in the uninjured condition (Roberts et al., 2013). Although the immunomodulation and trophic release of mesenchymal progenitors for regeneration command considerable attention (Caplan and Dennis, 2006; Le Blanc and Ringden, 2007), they also seem to play physiological roles in sustaining homeostasis of some tissues in normal conditions even without affecting the number and function of stem cells. Therefore, impairment of mesenchymal progenitors might directly contribute to the progression of aging. Mesenchymal stem cells/progenitors may be a subcategory of adult stem cells. However, in a physiological condition, at least in skeletal muscle, their contribution to generation of parenchymal cells is limited (Uezumi et al., 2010). Although contributions of bone marrow mesenchymal stem cells/progenitors to replacement of parenchymal cells have been reported, their trophic mediators seem to be the vital roles for the therapeutic effect (Caplan and Dennis, 2006). In addition, similar types of cells have been isolated from other adult tissues, including adipose, lung, skeletal muscle, salivary gland, and skin tissues (Zuk et al., 2002; Lama et al., 2007; Sudo et al., 2007; Uezumi et al., 2010). Under this criterion, mesenchymal stem/progenitors are a distinct type of stem cells from adult tissue-specific stem cells. Therefore, from this point of view, the concept of mesenchymal progenitors will be indispensable to understanding age-related alterations.

In this review, we will discuss the aged-related changes in stem cells that connect to the other theories of aging. In addition, we will discuss the possibility of a mesenchymal progenitor theory of aging based on recent research and speculations.

\section{ADULT TISSUE-SPECIFIC STEM CELLS AND MESENCHYMAL PROGENITOR CELLS}

Adult stem cells are defined as cells having both (multiple) differentiation and self-renewal potentials. They are indispensable for renewal and regeneration of parenchymal cells after damage. In adult mammals, many but not all tissues have functional resident tissue-specific stem cells that satisfy the criteria, including hematopoietic, skeletal muscle, pigment, epithelial, sperm, adipose, intestinal, and neural stem cells. Although a subset of adult stem cells is maintained in a quiescent state ( $\mathrm{Li}$ and Clevers, 2010), they show massive and repetitive proliferative potential in response to trauma or various stimulations. Adult stem cells exhibit global suppression of RNA polymerase II serine-2 phosphorylation, which triggers productive transcription elongation, mRNA processing, and release of mature mRNA; therefore, the transcription and translation states of adult stem cells seem to be relatively lower than those of proliferating cells (Freter et al., 2010). Even in these states, recent studies have demonstrated that a cell in a quiescent state is more likely to be in "active state" rather than in "passive state" (Cheung and Rando, 2013). In fact, canonical Notch signaling is commonly used to maintain some adult stem cells (melanocytes, neural, intestinal, and muscle stem cells), and its loss induces unusual cell cycling and/or expression of proliferative markers until eventually adult stem cell pools are exhausted (Moriyama et al., 2006; Imayoshi et al., 2010; Fukada et al., 2011, 2013; Pellegrinet et al., 2011; Bjornson et al., 2012; Mourikis et al., 2012). Although the types of Notch receptors are not yet fully understood, adult stem cells express certain Notch receptors (for example, muscle stem cells express Notch 1-3), and activated stem cells or more committed cells express the ligand (Mourikis et al., 2012; Kawaguchi et al., 2013). In this case, activated stem cells or more committed cells serve as niche cells to maintain adult stem cells. Niches are essential not only to maintain the stem cell pool, but also to regulate the cell fate of stem cells (Rompolas et al., 2013).

Like other types of niche cells, mesenchymal progenitors function in bone marrow. Mesenchymal stem cells or mesenchymal progenitors were first characterized in bone marrow, and most studies of mesenchymal progenitors are based on in vitro culturing. Notably, Morikawa et al. first demonstrated the prospective isolation of mesenchymal stem/progenitors in bone marrow (Morikawa et al., 2009a). In addition, our group isolated prospectively them and identified mesenchymal progenitors in murine skeletal muscle as the PDGFR $\alpha+$ cell fraction (Uezumi et al., 2010, 2011, 2014). Muscle mesenchymal progenitors are also positive for CD90 and Sca-1, but negative for CD31 and CD45. Murine bone marrow-derived mesenchymal stem cells (MSCs) are also positive for PDGFR $\alpha$ and Sca-1 (Morikawa et al., 2009a). Mesenchymal progenitors in muscle are not derived from the somites from which myogenic cells arise because they are not labeled by Myf5-Cre or Pax3-Cre in mice (Joe et al., 2010; Liu et al., 2012). Interestingly, a subset of mesenchymal stem cells in bone marrow is labeled in $\mathrm{PO}$-Cre mice, raising the possibility that the cells have their developmental origin in the neural crest (Takashima et al., 2007; Morikawa et al., 2009b).

Intriguingly, Roberts et al. demonstrated the essential roles of mesenchymal progenitors (they called them stromal cells) in the homeostasis of skeletal muscle and hematopoiesis (Roberts et al., 2013). They used fibroblast activation protein-a (FAP) as a marker of stromal cells of mesenchymal origin and inserted human diphtheria toxin receptor, firefly luciferase, and mCherry genes into mice under an FAP promoter. In these analyses, high FAP expression was observed in skeletal muscle, salivary glands, bone marrow, subcutaneous adipose, and skin. FAP+ cells coexpress PDGFR $\alpha, \mathrm{Sca}-1$, and CD90. Therefore, they seem to be the ideal cell as a mesenchymal progenitor. Mice depleted of FAP+ cells by injecting diphtheria toxin exhibited a loss of skeletal muscle mass and reduction of B-lymphopoiesis and erythropoiesis. Surprisingly, two ubiquitin ligases (Atrogin-1 and MuRF1) which are essential factors for inducing skeletal muscle atrophy were upregulated in stromal cell-depleted mice 3 days after diphtheria toxin injections. This result suggests that mesenchymal progenitors have direct roles in myofibers but not in muscle stem cells. Taken together, the roles of mesenchymal progenitors are diverse, and impairment of mesenchymal progenitors is a strong candidate in the impairment of tissues, including aging (Figure 1). In the next section, we will introduce evidence for a relationship between the theory to explain aging and adult stem cells and/or mesenchymal progenitors. 


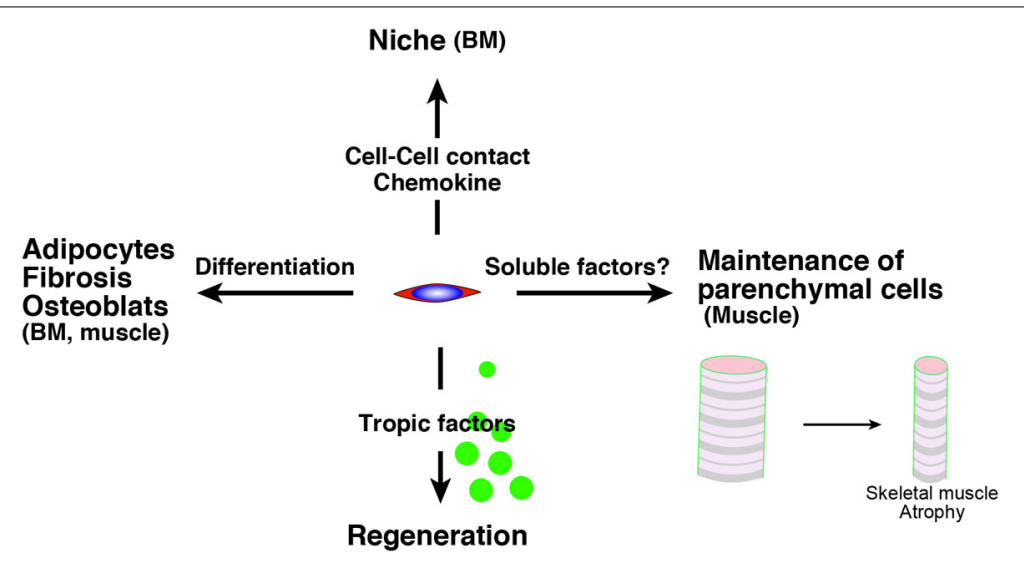

FIGURE 1 | The roles of mesenchymal progenitors. Mesenchymal progenitors have as least four aspects. (1) The differentiative potential into adipocytes, fibrosis-related cells, osteoblast, which are observed in BM and skeletal muscle. (2) They serve as niche cells in BM through the direct cell contact or chemokine. (3) They release trophic factors to promote tissue regeneration. (4) They sustain parenchymal cells (myofiber) in skeletal muscle via unknown mechanism. BM, bone marrow.

\section{OXIDATIVE STRESS AND STEM CELLS (FREE RADICAL THEORY AND STEM CELLS)}

Free radicals are unstable molecules that have an unpaired electron, and they include nitric oxide (NO), some of the reactive oxygen species (ROS), and their reactive products. ROS consist of superoxide anions $\left(\mathrm{O}_{2}^{-}\right)$, hydrogen peroxide $\left(\mathrm{H}_{2} \mathrm{O}_{2}\right)$, and hydroxyl radicals $(\mathrm{OH} \cdot)$, which are mainly generated in cells by the mitochondrial respiratory chain (Poyton et al., 2009). Extrinsic factors (radiation, ultraviolet light, and growth factors, among others) also cause ROS production. Oxidative stress is defined as an imbalance between production of free radicals/ROS and antioxidants (Reuter et al., 2010). Among antioxidants, superoxide dismutase (SOD), catalase, peroxiredoxin, thioredoxin, and glutathione systems are known as anti-oxidative enzymes, and nuclear factor erythroid 2-related factor 2 (NRF2) is known as a master regulator of these genes (Boutten et al., 2010), which belong to the cap" $n$ " collar (CNC) family, members of which have a conserved basic leucine zipper structure. NRF2 is regulated by KEAP1 (Kelch-like erythroid cell-derived protein with $\mathrm{CNC}$ homology $[\mathrm{ECH}]$-associated proteins), and ubiquitination of NRF2 by KEAP1 leads to degradation of NRF2 (Itoh et al., 1999).

Hochmuth et al. demonstrated that the regulation of ROS by Keap1 and Nrf2 controls intestinal stem cell proliferation in Drosophila (Hochmuth et al., 2011). In young flies, CncC (a homolog of NRF2) induces antioxidant genes, which result in low oxidative stress and maintain intestinal stem cells in the quiescent state. However, Keap1 suppressed the transcriptional activities of $\mathrm{CncC}$ in old flies and led to deceased expression of antioxidant genes, which resulted in a high ROS and proliferative condition leading to aged-related degeneration of the intestinal epithelium. On the other hand, Tsai et al. found that murine hematopoietic stem cells and progenitor pools were expanded in Nrf2-knockout mice, which indicates an intrinsic dysfunction of hematopoietic stem cells in their migration and retention in the niche (Tsai et al., 2013). Although the stem cell systems and species in the two studies differ, this system might be used in some stem cell systems across species.

Forkhead box O (FOXO) is another molecule that regulates the ROS pathway in hematopoietic stem cells. The FOXO subfamily (FOXO1, 3a, 4, and 6) is known as the downstream target of the PI3K-AKT signaling pathway. Mice conditionally depleted of Foxo1/3a/4 showed an increase in ROS and myeloid lineage expansion, lymphoid developmental abnormalities, and a decreased number of hematopoietic stem cells (Tothova et al., 2007). The authors also demonstrated that the antioxidant $\mathrm{N}$-acetyl cysteine (NAC) rescues a proportion of hematopoietic stem cells in Foxo1/3a/4-conditional KO mice. Miyamoto et al. observed that Foxo3a-null hematopoietic stem cells show increased ROS levels and decreased mRNA expression of Sod and catalase. The authors also demonstrated that aged Foxo3anull mice exhibited a loss of the hematopoietic stem cell pool, although young Foxo3a-null mice did not show such a defect (Miyamoto et al., 2007). FOXO3 is also essential in the nervous system to maintain neural stem cells (NSCs) (Renault et al., 2009). The depletion of Foxo3 leads to decreased number of neural stem cells in vivo. In addition, Foxo3-null NSCs exhibited decreased self-renewal and an impaired differentiative potential. Using Foxo1/3a/4-conditional KO mice, Paik et al. also revealed the essential roles of FOXOs in maintaining the quiescent state of neural stem cells and self-renewal. Foxo-null neural stem cells exhibited hyperproliferation and up-regulation of ROS level, but NAC treatment does not attenuate their proliferation (Paik et al., 2009). On the other hand, the decline in self-renewal of Foxo-null neural stem cells was rescued by NAC treatment. The importance of ROS levels in muscle stem cells is still unknown, but Pallafacchina et al. reported that quiescent muscle stem cells express antioxidant genes at a much higher level than proliferating myoblasts do (Pallafacchina et al., 2010). They also demonstrated that quiescent muscle stem cells are more resistant to hydrogen peroxide damage than proliferating myoblasts. These results imply that adult stem cells commonly have anti-oxidative 
stress pathways, the dysfunction of which occurs during the aging processes in adult stem cells. Taken together, stem cell compartments seem to be directly affected in the free radical theory, in which free radicals lead to or accelerate the aging process. In addition, FOXOs and NRF2 might be major master regulators that suppress ROS in adult stem cells across species.

Intriguingly, Foxos and Nrf2 are involved in the life span of C. elegans. Two genes were identified as responsible for longevity in C. elegans. One is the daf-2 gene, an equal homolog to both the mammalian insulin and IGF-1 receptors, and the other is age-1, a homolog to the worm PI3K-kinase catalytic subunit (Friedman and Johnson, 1988; Kenyon et al., 1993). In C. elegans, a mutation of either gene results in a profound extension of the lifespan. Insulin/IGF-1 signaling inhibits both DAF-16 (homolog of FOXO) and SKN-1 (homolog of NRF2) (Ogg et al., 1997; Tullet et al., 2008). Transgenically expressed SKN-1 prolongs the life span of $C$. elegans independently of DAF-16. Taken together, oxidative stress regulation by FOXOs and NRF2 is a conserved mechanism that contributes to the life span of stem cells and worms, and this system might be a common regulator for maintaining adult stem cells in mammalian tissues (Figure 2).

Hutchinson-Gilford progeria syndrome (HGPS) is an inherited disorder that causes premature aging and shortens the life span. The causative gene of HGPS is PROGERIN (PG), which is a truncated and farnesylated form of LAMIN A. HGPS affects mesenchymal linages. Zhang et al. produced iPS cells from HGPS

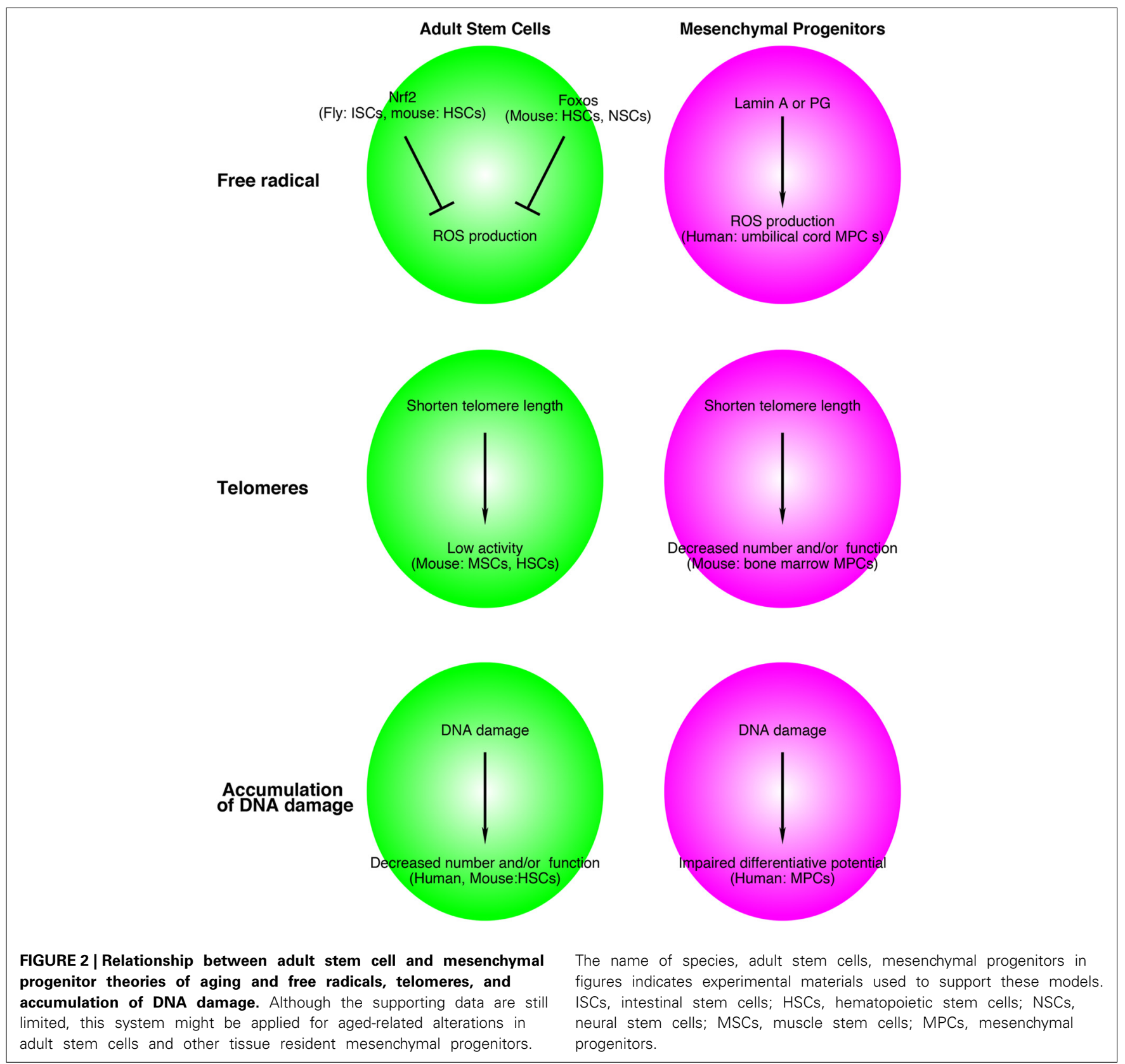


dermal fibroblasts and differentiated them into neural progenitors, endothelial cells, fibroblasts, vascular smooth muscle, and mesenchymal stem cells (Zhang et al., 2011b). Intriguingly, PG was not detected in control iPS cells, but patient iPS-derived mesenchymal stem cells express high levels of PG. Scaffidi and Misteli indicated that a similar mechanism occurs in normal aging (Scaffidi and Misteli, 2006). Similar to HGPS patient cells, aged fibroblasts showed accumulation of LAMIN A/C at the nuclear lamina, decreased expression of heterochromatin protein HP1 and LAP2, and increased DNA damage compared with young cells. Mateos et al. transduced LAMIN A or PG genes in human mesenchymal progenitors derived from umbilical cord and showed that LAMIN $A$ or $P G$ induced high levels of ROS in human mesenchymal stem cells (Figure 2). Lentiviral induction of $L A M I N A$ or $P G$ genes leads to impairment of chondrogenic differentiation, which partially depends on a decrease in manganese superoxide dismutase (MnSOD) and an increase of mitochondrial MnSOD-dependent ROS (Mateos et al., 2013). Taken together, ROS accumulation in mesenchymal stem cells/progenitors might contribute to the loss of stem cells and/or tissue homeostasis in HGPS and normal aging.

\section{TELOMERES AND STEM CELLS AND MESENCHYMAL PROGENITORS}

Characteristic repetitive DNA sequences and proteins in the end of eukaryotic chromosomes are called telomeres; telomeres are essential to maintain the stability of genomes. Telomere shortening is observed during aging of human fibroblasts (Harley et al., 1990). A DNA polymerase, named telomerase, induces telomere elongation. In order to elucidate the in vivo importance of telomere length, mice with the telomerase RNA component Terc knocked out $\left(\operatorname{Terc}^{-/-}\right)$were generated by Blasco et al. (1997). The mice show shortened telomere length, indicating that telomerase is essential to sustain telomere length. Early generations of Terc $^{-/-}$mice did not show severe phenotypes, but generation 6 (G6) Terc $^{-/-}$mice exhibited failures in highly proliferative organs including the hematopoietic and reproductive systems (Lee et al., 1998).

Deletion of an additional gene in $\mathrm{Terc}^{-/-}$mice produces significant changes in phenotype. For example, Wong et al. crossed $\operatorname{Terc}^{-/-}$mice with ataxia telangiectasia-mutated (Atm) knockout $\left(\mathrm{Atm}^{-/-}\right)$mice, and the offspring showed increased telomere erosion and genomic instability (Wong et al., 2003). Ataxiatelangiectasia results from mutation of the ATM gene and is characterized by accelerated telomere loss, genomic instability, progressive neurological degeneration, premature ageing, and increased incidence of neoplasia (Shiloh and Kastan, 2001). In addition, the frequency of T-cell lymphomas is suppressed in Atm and Terc double-knockout mice compared with $\mathrm{Atm}^{-/-}$mice, which have naturally occurring thymic lymphomas. Despite the cancer resistance of $\mathrm{Terc}^{-/-} \mathrm{Atm}^{-/-}$mice, their median life span is significantly shorter than that of $\mathrm{Terc}^{-/-} \mathrm{Atm}^{+/+}$mice.

Duchenne muscular dystrophy (DMD) is a well-known inherited X-linked disorder occurring in one in 3500 boys. The causative gene is DYSTROPHIN, which is essential for stability of the sarcolemma of myofibers. $M d x$ mice were discovered as mutants with high levels of muscle creatine kinase and pyruvate kinase in an inbred colony of C57BL/10 mice (Bulfield et al., 1984). Like humans, $m d x$ mice have a mutation in the dystrophin gene; therefore, the $m d x$ mouse is a model for human DMD. Although $m d x$ mice and DMD patients have the mutation in the same gene, the clinical and pathological phenotypes of $m d x$ mice are much milder than that of DMD patients. One of the differences is considered to be the great regenerative potential of muscle stem cells in $m d x$ mice (Fukada et al., 2010). Sacco et al. hypothesized that the longer telomere length of $m d x$ mice makes it possible for muscle stem cells to proliferate repeatedly, and generated $\operatorname{Terc}^{-/-} m d x$ mice ( $\left.m d x / \mathrm{mTR}\right)$. Compared with $m d x$ mice, $m d x / \mathrm{mTR}$ mice showed severe dystrophic phenotypes and decreased proliferative potential of muscle stem cells (Sacco et al., 2010). Unlike constitutively producing daughter cells such as hematopoietic stem cells, the proliferation of muscle stem cells during life is not very frequent. Therefore, the relationship between telomere length and aging of skeletal muscle is not clear, but these results indicate that the mechanism sustaining telomere length is one essential factor for retaining effective regeneration potential of skeletal muscles in the aged state.

As described above, many studies have shown the importance of telomere length to maintain stem cell function (Saeed and Iqtedar, 2013). In 2007, Ju et al. demonstrated that the environmental alterations due to the loss of Terc limit hematopoietic stem cell function and engraftment (Ju et al., 2007). The authors transplanted bone marrow cells from 2-month-old wild-type mice (CD45.1) into irradiated 2- and 12-month-old $\operatorname{Terc}^{-/-}$and $\operatorname{Terc}^{+/-}$littermates (CD45.2). First, they observed impaired B cell lymphopoiesis and increased myeloid proliferation in $\mathrm{Terc}^{-/-}$ recipient mice. Twelve-month-old $\mathrm{Terc}^{-/-}$mice showed a more severe defect of B cell lymphogenesis and accelerated myelopoiesis compared with 2-month-old $\mathrm{Terc}^{-/-}$mice. The environment of $\mathrm{Terc}^{-/-}$mice limited the engraftment of even wild-type hematopoietic stem cells. They also showed a shortened telomere length in mesenchymal progenitors (mesenchymal stromal cells: CD45-, TER119-, and CD31- adherent cells) in $\operatorname{Terc}^{-/-}$mice and a decrease in their number. Taken together, these results suggest that the shortening of telomere length in both stem cells and mesenchymal progenitors are factors of the telomere theory aging in various tissues (Figure 2).

\section{ACCUMULATION OF DNA DAMAGE AND TISSUE STEM CELLS AND MESENCHYMAL PROGENITORS}

DNA plays essential roles in maintaining almost all functions of the body by storing and transmitting genetic information. DNA damage generally leads to changes in normal DNA structure that results in a misreading of genetic information. Among diverse types of DNA damage, oxidative DNA damage, hydrolytic DNA damage, and ultraviolet and other radiation damages are regarded as the main causes. To avoid the accumulation of DNA damage, DNA repair pathways start to work and involve numerous enzymes in this complex process. According to many studies, an obstruction mutation of the DNA repair system causes symptoms of premature aging. In humans, a mutation of XPD (xeroderma pigmentosum group D) is known to cause a photosensitive form of the brittle hair disorder trichothiodystrophy. A study of de Boer at al. showed that mice with mutated $X p d$ genes $\left(X p d^{T T D}\right)$ exhibit 
many aged-related symptoms including osteoporosis, kyphosis, osteosclerosis, early graying, cachexia, infertility, and a reduced life span although the mice are born with developmentally normal phenotypes (de Boer et al., 2002). In addition, Rossi et al. showed that an Xpd deficiency did not result in the depletion of hematopoietic stem cells with age, but that the reconstitution and proliferative potential of hematopoietic stem cell was severely affected (Rossi et al., 2007).

The non-homologous end joining (NHEJ) pathway is a well known mechanism to repair DNA double-strand breaks. One of its components is DNA ligase IV. Using Lig4-deficient mice (Lig4 ${ }^{\text {Y288C }}$ mice), Nijnik found that impairment of the NHEJ pathway causes a progressive loss of hematopoietic stem cells during aging (Nijnik et al., 2007). Like XPD, loss of the hematopoietic stem cell pool was not observed, but functional defects of HSCs with age were reported in Ku80 (another NHEJ component)deficient mice (Rossi et al., 2007). In human hematopoietic stem cells and progenitors, DNA damage occurs during aging, and this accumulation of DNA is independent of telomere length (Rube et al., 2011). The relationships between DNA repair, aging, and mesenchymal progenitors remain to be elucidated. However, it has been reported at least that DNA-damaging agents such as hydrogen peroxide and paraquat lead to a loss of osteogenic differentiative potential (dexamethasone-induced ALP and mineralization) of human mesenchymal stromal cells (Alves et al., 2010) (Figure 2).

Accumulating studies also demonstrated that a component of serum is altered during aging. By utilizing parabiotic pairings, Conboy et al. showed that the age-related decline of muscle stem cell activity can be modulated by systemic factors that change with age (Conboy et al., 2005). Using young and aged ( $\sim 24$ months) mouse serum, same groups discovered that aged serum alters the cell fate of muscle stem cells (Brack et al., 2007). One of the candidates for the putative "aged serum" component is canonical Wnt-signaling-related molecules. Canonical Wnt signaling uses a $\beta$-catenin-dependent mechanism. Wnt signaling is an essential pathway for development and regeneration pathogenesis of many diseases, and accelerated Wnt activity is observed in different aged animal models including mice (Brack et al., 2007; Liu et al., 2007; Zhang et al., 2011a). Naito et al. identified complement $\mathrm{Clq}$ as a canonical activator of Wnt signaling that is upregulated in aged mice serum. They also showed that C1q can bind Frizzle (a receptor for Wnt) and activate canonical Wnt via cleavage of the ectodomain of the Wnt coreceptor LRP6 (lowdensity lipoprotein receptor-related protein 6) (Naito ET AL., 2012). Using rat bone marrow-derived mesenchymal stem cells, Zhang et al. demonstrated that Wnt/ $\beta$-catenin signaling induced the senescence or aging in mesenchymal stem cells that accompanies DNA damage (Zhang et al., 2011a). Therefore, acceleration of the canonical Wnt signaling pathway might affect both stem cell compartments and mesenchymal progenitors in aged tissues.

\section{PROSPECTS}

As described above, much evidence has shown that oxidative stress, telomere length, and DNA damage affect adult stem cells. The functions of mesenchymal progenitors are also affected by them. Oxidative stress, telomere length, and DNA damage, do not describe independent events, but they are interrelated phenomena. For example, oxidative stress leads to DNA damage (Kasai, 1997) and shortening of telomeres (von Zglinicki, 2002). Shortened telomere length in turn results in DNA damage (Vaziri and Benchimol, 1996). At least, mesenchymal progenitors serve as niche cell of hematopoietic stem cells. Therefore, the loss of mesenchymal progenitors leads to a decrease in stem cell function. In

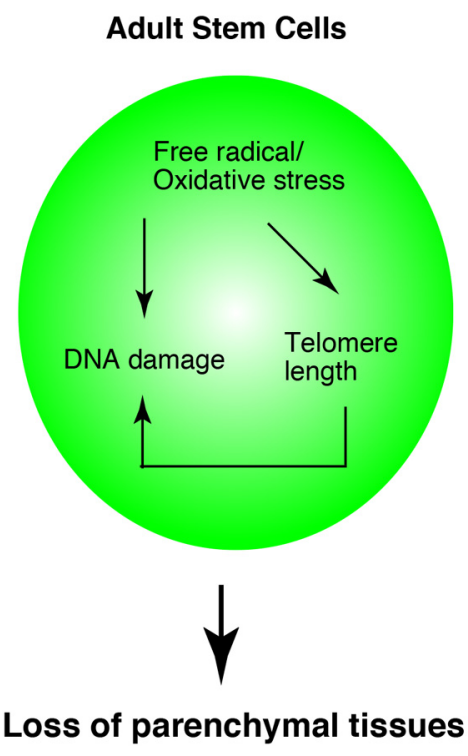

FIGURE 3 | Adult stem cell and mesenchymal progenitor theories of aging. Interrelationship of three theories in adult stem cells and mesenchymal progenitors. Mesenchymal progenitors serve as the niche cells for hematopoietic stem cells. In skeletal muscle, mesenchymal

\section{Mesenchymal Progenitors}

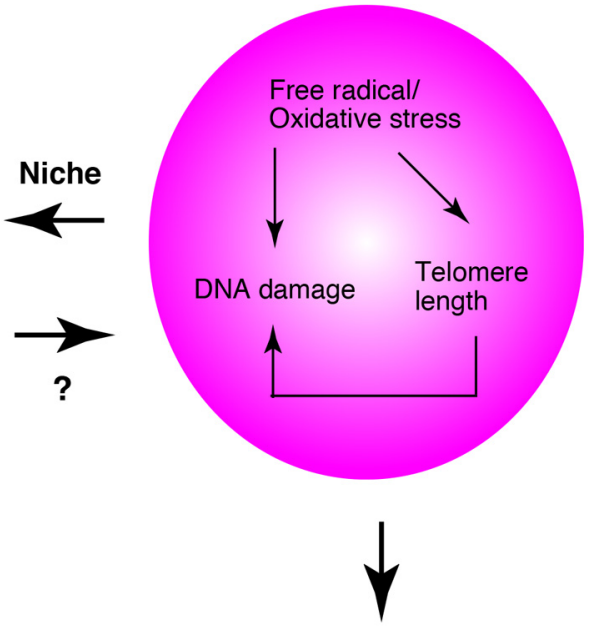

Loss of tissue homeostasis progenitors regulate myofibers directly. Therefore, this model is based on the studies of hematopoiesis and skeletal muscle system. The appropriateness of this system in other tissues remains to be elucidated. 
addition, dysfunction of mesenchymal progenitors might lead to a loss of tissue homeostasis without affecting adult stem cells, as was observed in skeletal muscle biology (Figure 3).

Cellular senescence is a unique state generally defined as irreversible cell cycle arrest. Recent studies have shown that senescent cells exhibit a robust increase in mRNA expression and secretion of numerous proinflammatory cytokines, which work in a paracrine manner (Campisi et al., 2011). Therefore, the chronic inflammation evoked by senescent cells is proposed as one causative factor of age-related degeneration. p16INK4a expression is increased in most senescent cells, and many studies showed that the age-dependent increase in p16INK4a expression is linked to impairment of the number and/or function of adult stem cells (hematopoietic, skeletal muscle, and nervous system) (Janzen et al., 2006; Molofsky et al., 2006; Sousa-Victor et al., 2014). Therefore cellular senescence is one mechanism of the age-related alteration of adult stem cells. In addition, p16INK4a expression induced senescence, and a knockdown of p16INK4a prevented the senescence of human mesenchymal stem cells (Shibata et al., 2007). Collectively, detrimental cellular senescence-mediated autocrine and paracrine effects contribute to "adult stem cell and mesenchymal progenitor theories of aging"; of course, it is possible that cellular senescence plays roles in differentiated cells rather than in adult stem cells and mesenchymal progenitors.

Fibroblasts can be observed in all tissues. Fibroblasts and mesenchymal progenitors show similar morphologies, but their differentiative potentials distinguish them. Sudo et al. investigated the differentiation potential of human fibroblasts derived from various tissues and found that cells originally considered fibroblasts have potential to differentiate into the mesenchymal lineage, which includes osteoblasts, chondrocytes, and adipocytes (Sudo et al., 2007). These results suggest that all tissues and organs contain mesenchymal progenitor cells. The identification of mesenchymal progenitor-derived factors and/or -expressing molecules might lead to development of anti-aging drugs that sustain the functions of aged tissues and organs. Collectively, in addition to stem cell biology, the investigation of tissue resident mesenchymal progenitors will be an essential project to sustain humans in a healthy condition for as long as possible.

\section{ACKNOWLEDGMENTS}

We thank Katherine Ono for comments on the manuscript. So-ichiro Fukada was supported by JSPS KAKENHI grant, Grantin Aid for Young Scientists (A) (25702044), Intramural Research Grant (22-5 and 25-5) for Neurological and Psychiatric Disorders of NCNP.

\section{REFERENCES}

Alves, H., Munoz-Najar, U., De Wit, J., Renard, A. J., Hoeijmakers, J. H., Sedivy, J. M., et al. (2010). A link between the accumulation of DNA damage and loss of multi-potency of human mesenchymal stromal cells. J. Cell. Mol. Med. 14, 2729-2738. doi: 10.1111/j.1582-4934.2009.00931.x

Bjornson, C. R., Cheung, T. H., Liu, L., Tripathi, P. V., Steeper, K. M., and Rando, T. A. (2012). Notch signaling is necessary to maintain quiescence in adult muscle stem cells. Stem Cells 30, 232-242. doi: 10.1002/stem.773

Blasco, M. A., Lee, H. W., Hande, M. P., Samper, E., Lansdorp, P. M., DePinho, R. A., et al. (1997). Telomere shortening and tumor formation by mouse cells lacking telomerase RNA. Cell 91, 25-34. doi: 10.1016/S0092-8674(01)80006-4
Bortoli, S., Renault, V., Eveno, E., Auffray, C., Butler-Browne, G., and Pietu, G. (2003). Gene expression profiling of human satellite cells during muscular aging using cDNA arrays. Gene 321, 145-154. doi: 10.1016/j.gene.2003.08.025

Boutten, A., Goven, D., Boczkowski, J., and Bonay, M. (2010). Oxidative stress targets in pulmonary emphysema: focus on the Nrf2 pathway. Exp. Opin. Ther. Targets 14, 329-346. doi: 10.1517/14728221003629750

Brack, A. S., Conboy, M. J., Roy, S., Lee, M., Kuo, C. J., Keller, C., et al. (2007). Increased Wnt signaling during aging alters muscle stem cell fate and increases fibrosis. Science 317, 807-810. doi: 10.1126/science.1144090

Bulfield, G., Siller, W. G., Wight, P. A., and Moore, K. J. (1984). X chromosomelinked muscular dystrophy $(\mathrm{mdx})$ in the mouse. Proc. Natl. Acad. Sci. U.S.A. 81, 1189-1192. doi: 10.1073/pnas.81.4.1189

Campisi, J., Andersen, J. K., Kapahi, P., and Melov, S. (2011). Cellular senescence: a link between cancer and age-related degenerative disease? Sem. Cancer Biol. 21, 354-359. doi: 10.1016/j.semcancer.2011.09.001

Caplan, A. I., and Dennis, J. E. (2006). Mesenchymal stem cells as trophic mediators. J. Cell Biochem. 98, 1076-1084. doi: 10.1002/jcb.20886

Cheung, T. H., and Rando, T. A. (2013). Molecular regulation of stem cell quiescence. Nat. Rev. Mol. Cell Biol. 14, 329-340. doi: 10.1038/nrm3591

Christov, C., Chretien, F., Abou-Khalil, R., Bassez, G., Vallet, G., Authier, F. J., et al. (2007). Muscle satellite cells and endothelial cells: close neighbors and privileged partners. Mol. Biol. Cell 18, 1397-1409. doi: 10.1091/mbc.E06-08-0693

Collins, C. A., Zammit, P. S., Ruiz, A. P., Morgan, J. E., and Partridge, T. A. (2007). A population of myogenic stem cells that survives skeletal muscle aging. Stem Cells 25, 885-894. doi: 10.1634/stemcells.2006-0372

Conboy, I. M., Conboy, M. J., Wagers, A. J., Girma, E. R., Weissman, I. L., and Rando, T. A. (2005). Rejuvenation of aged progenitor cells by exposure to a young systemic environment. Nature 433, 760-764. doi: 10.1038/nature 03260

de Boer, J., Andressoo, J. O., de Wit, J., Huijmans, J., Beems, R. B., van Steeg, H., et al. (2002). Premature aging in mice deficient in DNA repair and transcription. Science 296, 1276-1279. doi: 10.1126/science.1070174

Freter, R., Osawa, M., and Nishikawa, S. (2010). Adult stem cells exhibit global suppression of RNA polymerase II serine-2 phosphorylation. Stem Cells 28, 1571-1580. doi: 10.1002/stem.476

Friedman, D. B., and Johnson, T. E. (1988). A mutation in the age-1 gene in Caenorhabditis elegans lengthens life and reduces hermaphrodite fertility. Genetics 118, 75-86.

Fukada, S., Ma, Y., Ohtani, T., Watanabe, Y., Murakami, S., and Yamaguchi, M. (2013). Isolation, characterization, and molecular regulation of muscle stem cells. Front. Physiol. 4:317. doi: 10.3389/fphys.2013.00317

Fukada, S., Morikawa, D., Yamamoto, Y., Yoshida, T., Sumie, N., Yamaguchi, M., et al. (2010). Genetic background affects properties of satellite cells and $\mathrm{mdx}$ phenotypes. Am. J. Pathol. 176, 2414-2424. doi: 10.2353/ajpath.2010.090887

Fukada, S., Yamaguchi, M., Kokubo, H., Ogawa, R., Uezumi, A., Yoneda, T., et al. (2011). Hesr1 and Hesr3 are essential to generate undifferentiated quiescent satellite cells and to maintain satellite cell numbers. Development 138, 4609-4619. doi: 10.1242/dev.067165

Harley, C. B., Futcher, A. B., and Greider, C. W. (1990). Telomeres shorten during ageing of human fibroblasts. Nature 345, 458-460. doi: 10.1038/345458a0

Harman, D. (1956). Aging: a theory based on free radical and radiation chemistry. J. Gerontol. 11, 298-300. doi: 10.1093/geronj/11.3.298

Hochmuth, C. E., Biteau, B., Bohmann, D., and Jasper, H. (2011). Redox regulation by Keap1 and Nrf2 controls intestinal stem cell proliferation in Drosophila. Cell Stem Cell 8, 188-199. doi: 10.1016/j.stem.2010.12.006

Imayoshi, I., Sakamoto, M., Yamaguchi, M., Mori, K., and Kageyama, R. (2010). Essential roles of Notch signaling in maintenance of neural stem cells in developing and adult brains. J. Neurosci. 30, 3489-3498. doi: 10.1523/JNEUROSCI.4987-09.2010

Itoh, K., Wakabayashi, N., Katoh, Y., Ishii, T., Igarashi, K., Engel, J. D., et al. (1999). Keap1 represses nuclear activation of antioxidant responsive elements by Nrf2 through binding to the amino-terminal Neh2 domain. Genes Dev. 13, 76-86. doi: 10.1101/gad.13.1.76

Janzen, V., Forkert, R., Fleming, H. E., Saito, Y., Waring, M. T., Dombkowski, D. M., et al. (2006). Stem-cell ageing modified by the cyclin-dependent kinase inhibitor p16INK4a. Nature 443, 421-426. doi: 10.1038/nature05159

Joe, A. W., Yi, L., Natarajan, A., Le Grand, F., So, L., Wang, J., et al. (2010). Muscle injury activates resident fibro/adipogenic progenitors that facilitate myogenesis. Nat. Cell Biol. 12, 153-163. doi: 10.1038/ncb2015 
Ju, Z., Jiang, H., Jaworski, M., Rathinam, C., Gompf, A., Klein, C., et al. (2007). Telomere dysfunction induces environmental alterations limiting hematopoietic stem cell function and engraftment. Nat. Med. 13, 742-747. doi: $10.1038 / \mathrm{nm} 1578$

Kadi, F., Charifi, N., Denis, C., and Lexell, J. (2004). Satellite cells and myonuclei in young and elderly women and men. Muscle Nerve 29, 120-127. doi: $10.1002 /$ mus. 10510

Kasai, H. (1997). Analysis of a form of oxidative DNA damage, 8-hydroxy-2' deoxyguanosine, as a marker of cellular oxidative stress during carcinogenesis. Mutation research 387, 147-163. doi: 10.1016/S1383-5742(97)00035-5

Kawaguchi, D., Furutachi, S., Kawai, H., Hozumi, K., and Gotoh, Y. (2013). Dll1 maintains quiescence of adult neural stem cells and segregates asymmetrically during mitosis. Nat. Commun. 4, 1880. doi: 10.1038/ncomms2895

Kenyon, C., Chang, J., Gensch, E., Rudner, A., and Tabtiang, R. (1993). A C. elegans mutant that lives twice as long as wild type. Nature 366, 461-464. doi: $10.1038 / 366461 \mathrm{a0}$

Kiel, M. J., Yilmaz, O. H., Iwashita, T., Yilmaz, O. H., Terhorst, C., and Morrison, S. J. (2005). SLAM family receptors distinguish hematopoietic stem and progenitor cells and reveal endothelial niches for stem cells. Cell 121, 1109-1121. doi: 10.1016/j.cell.2005.05.026

Lama, V. N., Smith, L., Badri, L., Flint, A., Andrei, A. C., Murray, S., et al. (2007). Evidence for tissue-resident mesenchymal stem cells in human adult lung from studies of transplanted allografts. J. Clin. Invest. 117, 989-996. doi: $10.1172 / \mathrm{JCI} 29713$

Le Blanc, K., and Ringden, O. (2007). Immunomodulation by mesenchymal stem cells and clinical experience. J. Int. Med. 262, 509-525. doi: 10.1111/j.13652796.2007.01844.x

Lee, H. W., Blasco, M. A., Gottlieb, G. J., Horner, J. W. 2nd., Greider, C. W., and DePinho, R. A. (1998). Essential role of mouse telomerase in highly proliferative organs. Nature 392, 569-574. doi: 10.1038/33345

Li, L., and Clevers, H. (2010). Coexistence of quiescent and active adult stem cells in mammals. Science 327, 542-545. doi: 10.1126/science. 1180794

Linton, P. J., and Dorshkind, K. (2004). Age-related changes in lymphocyte development and function. Nat. Immunol. 5, 133-139. doi: 10.1038/ni1033

Liu, H., Fergusson, M. M., Castilho, R. M., Liu, J., Cao, L., Chen, J., et al. (2007). Augmented Wnt signaling in a mammalian model of accelerated aging. Science 317, 803-806. doi: 10.1126/science.1143578

Liu, W., Liu, Y., Lai, X., and Kuang, S. (2012). Intramuscular adipose is derived from a non-Pax3 lineage and required for efficient regeneration of skeletal muscles. Dev. Biol. 361, 27-38. doi: 10.1016/j.ydbio.2011.10.011

Mateos, J., De la Fuente, A., Lesende-Rodriguez, I., Fernandez-Pernas, P., Arufe, M. C., and Blanco, F. J. (2013). Lamin A deregulation in human mesenchymal stem cells promotes an impairment in their chondrogenic potential and imbalance in their response to oxidative stress. Stem Cell Res. 11, 1137-1148. doi: 10.1016/j.scr.2013.07.004

Miyamoto, K., Araki, K. Y., Naka, K., Arai, F., Takubo, K., Yamazaki, S., et al. (2007). Foxo3a is essential for maintenance of the hematopoietic stem cell pool. Cell Stem Cell 1, 101-112. doi: 10.1016/j.stem.2007.02.001

Molofsky, A. V., Slutsky, S. G., Joseph, N. M., He, S., Pardal, R., Krishnamurthy, J., et al. (2006). Increasing pl6INK4a expression decreases forebrain progenitors and neurogenesis during ageing. Nature 443, 448-452. doi: 10.1038/nature05091

Morikawa, S., Mabuchi, Y., Kubota, Y., Nagai, Y., Niibe, K., Hiratsu, E., et al. (2009a). Prospective identification, isolation, and systemic transplantation of multipotent mesenchymal stem cells in murine bone marrow. J. Exp. Med. 206 , 2483-2496. doi: 10.1084/jem.20091046

Morikawa, S., Mabuchi, Y., Niibe, K., Suzuki, S., Nagoshi, N., Sunabori, T., et al. (2009b). Development of mesenchymal stem cells partially originate from the neural crest. Biochem. Biophys. Res. Commun. 379, 1114-1119. doi: 10.1016/j.bbrc.2009.01.031

Moriyama, M., Osawa, M., Mak, S. S., Ohtsuka, T., Yamamoto, N., Han, H., et al. (2006). Notch signaling via Hes1 transcription factor maintains survival of melanoblasts and melanocyte stem cells. J. Cell Biol. 173, 333-339. doi: $10.1083 /$ jcb. 200509084

Morrison, S. J., Wandycz, A. M., Akashi, K., Globerson, A., and Weissman, I. L. (1996). The aging of hematopoietic stem cells. Nat. Med. 2, 1011-1016. doi: 10.1038/nm0996-1011

Mourikis, P., Sambasivan, R., Castel, D., Rocheteau, P., Bizzarro, V., and Tajbakhsh, S. (2012). A critical requirement for notch signaling in maintenance of the quiescent skeletal muscle stem cell state. Stem Cells 30, 243-252. doi: 10.1002/stem.775

Naito, A. T., Sumida, T., Nomura, S., Liu, M. L., Higo, T., Nakagawa, A., et al. (2012). Complement Clq activates canonical Wnt signaling and promotes aging-related phenotypes. Cell 149, 1298-1313. doi: 10.1016/j.cell.2012.03.047

Nijnik, A., Woodbine, L., Marchetti, C., Dawson, S., Lambe, T., Liu, C., et al. (2007). DNA repair is limiting for haematopoietic stem cells during ageing. Nature 447, 686-690. doi: 10.1038/nature05875

Nishimura, E. K. (2011). Melanocyte stem cells: a melanocyte reservoir in hair follicles for hair and skin pigmentation. Pigment Cell Melanoma Res. 24, 401-410. doi: 10.1111/j.1755-148X.2011.00855.x

Nishimura, E. K., Granter, S. R., and Fisher, D. E. (2005). Mechanisms of hair graying: incomplete melanocyte stem cell maintenance in the niche. Science 307, 720-724. doi: 10.1126/science.1099593

Ogg, S., Paradis, S., Gottlieb, S., Patterson, G. I., Lee, L., Tissenbaum, H. A., et al. (1997). The Fork head transcription factor DAF-16 transduces insulinlike metabolic and longevity signals in C. elegans. Nature 389, 994-999. doi: $10.1038 / 40194$

Omatsu, Y., Sugiyama, T., Kohara, H., Kondoh, G., Fujii, N., Kohno, K., et al. (2010). The essential functions of adipo-osteogenic progenitors as the hematopoietic stem and progenitor cell niche. Immunity 33, 387-399. doi: 10.1016/j.immuni.2010.08.017

Paik, J. H., Ding, Z., Narurkar, R., Ramkissoon, S., Muller, F., Kamoun, W. S., et al. (2009). FoxOs cooperatively regulate diverse pathways governing neural stem cell homeostasis. Cell Stem Cell 5, 540-553. doi: 10.1016/j.stem.2009.09.013

Pallafacchina, G., Francois, S., Regnault, B., Czarny, B., Dive, V., Cumano, A., et al. (2010). An adult tissue-specific stem cell in its niche: a gene profiling analysis of in vivo quiescent and activated muscle satellite cells. Stem Cell Res. 4, 77-91. doi: 10.1016/j.scr.2009.10.003

Pan, L., Chen, S., Weng, C., Call, G., Zhu, D., Tang, H., et al. (2007). Stem cell aging is controlled both intrinsically and extrinsically in the Drosophila ovary. Cell Stem Cell 1, 458-469. doi: 10.1016/j.stem.2007.09.010

Pang, W. W., Price, E. A., Sahoo, D., Beerman, I., Maloney, W. J., Rossi, D. J., et al. (2011). Human bone marrow hematopoietic stem cells are increased in frequency and myeloid-biased with age. Proc. Natl. Acad. Sci. U.S.A. 108, 20012-20017. doi: 10.1073/pnas.1116110108

Pellegrinet, L., Rodilla, V., Liu, Z., Chen, S., Koch, U., Espinosa, L., et al. (2011). Dll1- and dll4-mediated notch signaling are required for homeostasis of intestinal stem cells. Gastroenterology 140, 1230-1240.e1-7. doi: 10.1053/j.gastro.2011.01.005

Poyton, R. O., Ball, K. A., and Castello, P. R. (2009). Mitochondrial generation of free radicals and hypoxic signaling. Trends Endocrinol. Metab. 20, 332-340. doi: 10.1016/j.tem.2009.04.001

Renault, V. M., Rafalski, V. A., Morgan, A. A., Salih, D. A., Brett, J. O., Webb, A. E., et al. (2009). FoxO3 regulates neural stem cell homeostasis. Cell Stem Cell 5, 527-539. doi: 10.1016/j.stem.2009.09.014

Reuter, S., Gupta, S. C., Chaturvedi, M. M., and Aggarwal, B. B. (2010). Oxidative stress, inflammation, and cancer: how are they linked? Free Radic. Biol. Med. 49, 1603-1616. doi: 10.1016/j.freeradbiomed.2010.09.006

Roberts, E. W., Deonarine, A., Jones, J. O., Denton, A. E., Feig, C., Lyons, S. K., et al. (2013). Depletion of stromal cells expressing fibroblast activation protein-alpha from skeletal muscle and bone marrow results in cachexia and anemia. J. Exp. Med. 210, 1137-1151. doi: 10.1084/jem.20122344

Rompolas, P., Mesa, K. R., and Greco, V. (2013). Spatial organization within a niche as a determinant of stem-cell fate. Nature 502, 513-518. doi: 10.1038 /nature12602

Rossi, D. J., Bryder, D., Seita, J., Nussenzweig, A., Hoeijmakers, J., and Weissman, I. L. (2007). Deficiencies in DNA damage repair limit the function of haematopoietic stem cells with age. Nature 447, 725-729. doi: 10.1038/nature05862

Rube, C. E., Fricke, A., Widmann, T. A., Furst, T., Madry, H., Pfreundschuh, M., et al. (2011). Accumulation of DNA damage in hematopoietic stem and progenitor cells during human aging. PLoS ONE 6:e17487. doi: 10.1371/journal.pone. 0017487

Sacco, A., Mourkioti, F., Tran, R., Choi, J., Llewellyn, M., Kraft, P., et al. (2010) Short telomeres and stem cell exhaustion model Duchenne muscular dystrophy in mdx/mTR mice. Cell 143, 1059-1071. doi: 10.1016/j.cell.2010.11.039

Saeed, H., and Iqtedar, M. (2013). Stem cell function and maintenance - ends that matter: role of telomeres and telomerase. J. Biosci. 38, 641-649. doi: $10.1007 / \mathrm{s} 12038-013-9346-3$ 
Scaffidi, P., and Misteli, T. (2006). Lamin A-dependent nuclear defects in human aging. Science 312, 1059-1063. doi: 10.1126/science.1127168

Shibata, K. R., Aoyama, T., Shima, Y., Fukiage, K., Otsuka, S., Furu, M., et al. (2007). Expression of the p16INK4A gene is associated closely with senescence of human mesenchymal stem cells and is potentially silenced by DNA methylation during in vitro expansion. Stem Cells 25, 2371-2382. doi: 10.1634/stemcells.2007-0225

Shiloh, Y., and Kastan, M. B. (2001). ATM: genome stability, neuronal development, and cancer cross paths. Adv. Cancer Res. 83, 209-254. doi: 10.1016/S0065230X(01)83007-4

Sousa-Victor, P., Gutarra, S., Garcia-Prat, L., Rodriguez-Ubreva, J., Ortet, L., Ruiz-Bonilla, V., et al. (2014). Geriatric muscle stem cells switch reversible quiescence into senescence. Nature 50, 316-321. doi: 10.1038/ nature 13013

Sudo, K., Kanno, M., Miharada, K., Ogawa, S., Hiroyama, T., Saijo, K., et al. (2007). Mesenchymal progenitors able to differentiate into osteogenic, chondrogenic, and/or adipogenic cells in vitro are present in most primary fibroblastlike cell populations. Stem Cells 25, 1610-1617. doi: 10.1634/stemcells. 2006-0504

Takashima, Y., Era, T., Nakao, K., Kondo, S., Kasuga, M., Smith, A. G., et al. (2007). Neuroepithelial cells supply an initial transient wave of MSC differentiation. Cell 129, 1377-1388. doi: 10.1016/j.cell.2007.04.028

Tavazoie, M., Van der Veken, L., Silva-Vargas, V., Louissaint, M., Colonna, L., Zaidi, B., et al. (2008). A specialized vascular niche for adult neural stem cells. Cell Stem Cell 3, 279-288. doi: 10.1016/j.stem.2008.07.025

Tothova, Z., Kollipara, R., Huntly, B. J., Lee, B. H., Castrillon, D. H., Cullen, D. E., et al. (2007). FoxOs are critical mediators of hematopoietic stem cell resistance to physiologic oxidative stress. Cell 128, 325-339. doi: 10.1016/j.cell.2007. 01.003

Tsai, J. J., Dudakov, J. A., Takahashi, K., Shieh, J. H., Velardi, E., Holland, A. M., et al. (2013). Nrf2 regulates haematopoietic stem cell function. Nat. Cell Biol. 15, 309-316. doi: 10.1038/ncb2699

Tullet, J. M., Hertweck, M., An, J. H., Baker, J., Hwang, J. Y., Liu, S., et al. (2008). Direct inhibition of the longevity-promoting factor SKN-1 by insulinlike signaling in C. elegans. Cell 132, 1025-1038. doi: 10.1016/j.cell.2008. 01.030

Uezumi, A., Fukada, S., Yamamoto, N., Takeda, S., and Tsuchida, K. (2010). Mesenchymal progenitors distinct from satellite cells contribute to ectopic fat cell formation in skeletal muscle. Nat. Cell Biol. 12, 143-152. doi: $10.1038 /$ ncb2014

Uezumi, A., Ikemoto-Uezumi, M., and Tsuchida, K. (2014). Roles of nonmyogenic mesenchymal progenitors in pathogenesis and regeneration of skeletal muscle. Front. Physiol. 5:68. doi: 10.3389/fphys.2014.00068
Uezumi, A., Ito, T., Morikawa, D., Shimizu, N., Yoneda, T., Segawa, M., et al. (2011). Fibrosis and adipogenesis originate from a common mesenchymal progenitor in skeletal muscle. J. Cell Sci. 124, 3654-3664. doi: 10.1242/jcs.086629

Vaziri, H., and Benchimol, S. (1996). From telomere loss to p53 induction and activation of a DNA-damage pathway at senescence: the telomere loss/DNA damage model of cell aging. Exp. Gerontol. 31, 295-301. doi: 10.1016/05315565(95)02025-X

von Zglinicki, T. (2002). Oxidative stress shortens telomeres. Trends Biochem. Sci. 27, 339-344. doi: 10.1016/S0968-0004(02)02110-2

Wong, K. K., Maser, R. S., Bachoo, R. M., Menon, J., Carrasco, D. R., Gu, Y., et al. (2003). Telomere dysfunction and Atm deficiency compromises organ homeostasis and accelerates ageing. Nature 421, 643-648. doi: 10.1038/ nature 01385

Yoshida, S., Sukeno, M., and Nabeshima, Y. (2007). A vasculature-associated niche for undifferentiated spermatogonia in the mouse testis. Science 317, 1722-1726. doi: 10.1126/science. 1144885

Zhang, D. Y., Wang, H. J., and Tan, Y. Z. (2011a). Wnt/beta-catenin signaling induces the aging of mesenchymal stem cells through the DNA damage response and the p53/p21 pathway. PLOS ONE 6:e21397. doi: 10.1371/journal.pone.0021397

Zhang, J., Lian, Q., Zhu, G., Zhou, F., Sui, L., Tan, C., et al. (2011b). A human iPSC model of Hutchinson Gilford Progeria reveals vascular smooth muscle and mesenchymal stem cell defects. Cell Stem Cell 8, 31-45. doi: 10.1016/j.stem.2010.12.002

Zuk, P. A., Zhu, M., Ashjian, P., De Ugarte, D. A., Huang, J. I., Mizuno, H., et al. (2002). Human adipose tissue is a source of multipotent stem cells. Mol. Biol. Cell 13, 4279-4295. doi: 10.1091/mbc.E02-02-0105

Conflict of Interest Statement: The authors declare that the research was conducted in the absence of any commercial or financial relationships that could be construed as a potential conflict of interest.

Received: 14 January 2014; accepted: 12 March 2014; published online: 28 March 2014. Citation: Fukada S, Ma Y and Uezumi A (2014) Adult stem cell and mesenchymal progenitor theories of aging. Front. Cell Dev. Biol. 2:10. doi: 10.3389/fcell.2014.00010 This article was submitted to Stem Cell Research, a section of the journal Frontiers in Cell and Developmental Biology.

Copyright (c) 2014 Fukada, Ma and Uezumi. This is an open-access article distributed under the terms of the Creative Commons Attribution License (CC BY). The use, distribution or reproduction in other forums is permitted, provided the original author(s) or licensor are credited and that the original publication in this journal is cited, in accordance with accepted academic practice. No use, distribution or reproduction is permitted which does not comply with these terms. 